\title{
Misused Terms in Linguistics
}

\author{
Evelina Leivada
}

$\mathrm{T}$

HE EVOlutionaRY BIOLOGist Eörs Szathmáry observed that linguists "would rather share each other's toothbrush than each other's terminology." This is far from an isolated view. Peter Hagoort, an eminent cognitive neuroscientist, voiced similar concerns.

When even at major linguistics conferences the program contains presentation[s] such as "A short tour through the minefield of linguistic terminology," one should realize that this state of affairs is a serious threat to the influence that linguists exert on the research agenda of cognitive science broadly. ${ }^{2}$

Tanja Kupisch and Jason Rothman, psycholinguists working primarily on bilingual development, recently noted that "[o]nce offered to the public domain, terminology can have far-reaching and long-lasting effects, even-perhaps especially-when these are unintended by their original promoters."

An effort to improve the terminological clarity and coherence of theoretical and experimental linguistics is long overdue. In this respect, linguists might consider following the lead of psychologists in identifying and discussing lists of inaccurate, ambiguous, misused, and polysemous terms. ${ }^{4}$ The focus throughout should be on key notions of the field. Terms such as feature, parameter, (grammaticality/acceptability) judgment, (language) universal, and Universal Grammar are omnipresent in linguistics. ${ }^{5}$ These notions are fundamental to the discipline and their misuse has important implications, not only for the coherence of the field, but also for its standing in the broader context of cognitive science. The following terms do not all satisfy the same criteria of inaccuracy, ambiguity, and misuse. The degrees to which they exhibit these characteristics vary, and this is part of the problem. This review will not focus solely on the conceptual clarity of these ten terms, but also on their inconsistent usage.

\section{Universal Grammar and Language Universals}

In Noam Chomsky's work, Universal Grammar (UG) is the source of our innate ability to acquire and use a natural language. ${ }^{6}$ The faculty of language consists of certain principles that are innate, common to our species, and available prior to any systematic exposure to a given language. ${ }^{7} \mathrm{UG}$ is sometimes identified with linguistic universals; but this is a mistake. When Chomsky talks about language universals, he does not refer to properties that are universally attested to in all languages, but to computational properties of the mind that are universal because they arise from a species-universal innate ability. ${ }^{8} \mathrm{~A}$ property $\mathrm{P}$ in a given language can reflect a universal computational principle even if $\mathrm{P}$ is not attested to in another language. ${ }^{9}$

There is no reason to assume that linguistic universals, understood as properties that are shared across languages, are necessarily derived from UG. Although most languages settle on a consistent word order, ${ }^{10}$ this preference does not reflect the imperatives of UG. Infants process and reshape input in a way that promotes the regularization of harmonic patterns. ${ }^{11}$ In fact, as Chomsky has argued, language development is guided by principles of general cognition, which work together with linguistic experience and UG. ${ }^{12}$ Given that a complete list of all the UG principles has not been compiled, the possibility that these principles are, for the most part, not language-specific, but have cognitive, third factor roots, cannot be ruled out.

\section{Parameter}

In its early days, UG was thought to consist of principles, which are invariant across languages, and a finite set of open parameters that are fixed by experience. The head-directionality parameter, for example, determines the position of the defining element of a phrase in relation to its complements. Some languages such as English are head-initial; others, such as Japanese, head-final. Setting one parameter enables predictions about the others. The original aim was to capture the panorama of cross-linguistic variation while positing as few parameters as possible. ${ }^{13}$ How many are a few? Twenty? Eighty? 1,534? $1,465,462$ ? Nobody has an answer to this question, and I suspect nobody ever will. Over the last two decades, the term "parameter" has been applied to every possible point 
of variation across languages, eventually giving rise to parameters of various sizes and domains of predictionmacroparameters, mesoparameters, and nanoparameters. This terminological fluidity is not innocent. Apart from having stripped the notion of a parameter of its original substance, it endowed the term with various custom-made definitions and objects of inquiry. Anybody can postulate a new nanoparameter, even on the basis of one lexical item. In such cases, the term "parameter" is still used despite it no longer carrying the same crucial implications as in its original context. Microparameters, for instance, make no robust, whole-grammar predictions of setability. Yet we cannot afford to dispose of these predictions. The notion that UG consists of parameters, which provide a guide into the variation space, relied on these predictions. One cannot simply use the term "parameter," or variants of the term, with an arbitrary meaning.

Terminological fluidity is not without consequences. In the case of the term "parameter," its misuse over the years raised serious concerns about the biological plausibility of an innate endowment for language that consists of millions of minimal points of variation. "If the number of parameters," Frederick Newmeyer observed, "needed to handle the different grammars of the world's languages, dialects, and (possibly) idiolects is in the thousands (or, worse, millions), then ascribing them to an innate UG to my mind loses all semblance of plausibility."14

\section{Feature}

If parameters come with various definitions and are often custom made, so are features. "Custom made" refers to proposals of the following form: feature A is proposed for language B on the basis of an item/structure C. In such proposals, the postulated primitive is purely data driven and designed specifically to fit a portion of the data. ${ }^{15}$ As partial descriptions of linguistic objects, ${ }^{16}$ features can be anything from roundedness in phonology to the extended projection principle (EPP) in syntax. ${ }^{17}$ These are both very different. Calling almost anything a feature creates both disciplinary and interdisciplinary concerns.

Where do features come from? For some scholars, the answer is UG itself: "The study of the feature inventory of UG requires a massive database compiled on the basis of detailed studies of particular grammars."18 Ascribing an inordinate number of primitives to $\mathrm{UG}$ is not an innocent move. It should not be a desirable move either, because this would mean that the basic atoms of linguistics are not derived, but postulated as innate. Linguists propose new features at will, but there is no theory of UG that can explain the existence of the requisite number of primitives. How many exactly? Again, there is no answer, because anything can be a partial description of a linguistic object. As Norbert Hornstein has pointed out, the real problem is that we have no hint of a theory of features.
Postulating the existence of a feature $\mathrm{A}$ in order to explain a structure B does not mean that we have shed light on our biological ability for language. ${ }^{19}$ Such inventions come at a high cost. ${ }^{20}$ Both field-internal terminological coherence and field-external visibility are diminished as a result. Using the same term to talk about very different things inevitably reduces internal coherence and consistency. Meanwhile, a biologist would have a hard time understanding what kind of primitives are classified as features in linguistics. This is problematic under the view that features are part of UG-our biological endowment for language. One could, of course, say that "features are properties of syntactic atoms," whereby "a feature [plural] for example is used analogously to chemists' use of $\mathrm{H}$ for the real-world thing hydrogen."21 This analogy is unpersuasive. The definition of a chemical element is specific and unambiguous, made exclusively on the basis of the number of protons within its nucleus. But linguists use the EPP in different ways across research groups. ${ }^{22}$ Additionally, we know exactly how many chemical elements have been discovered or created: 118. We can and do keep a count. There is a consensus that this is the right number; it is not a matter of one's definition or viewpoint. The same claim cannot be made for features.

\section{The Linguistic Genotype}

Linguists often talk about the genetic endowment for language; and this suggests that a part of the human genome is dedicated to language. There is no dispute that our species is biologically language-ready. This does not justify the claim that a portion of our genome is dedicated to language. According to Ana Villar and Antoni Gomila:

While it is obvious that any human mental capability requires a genetic make-up as long as it is a biological phenomenon, it cannot be simply assumed that there is going to be some part of the genome specialized for every mental faculty, or for language in particular. Not only because genes can be pleiotropic (i.e., involved in very different processes), but also because they don't work in isolation. ${ }^{23}$

A direct link between genetic makeup and linguistic phenotypic traits is untenable. First, genes do not code for grammatical properties. Second, a set of genes can affect multiple, potentially unrelated, phenotypes so that no direct, one-to-one relation exists between genes and phenotypic traits. Third, genes are not the only players in ontogenetic processes. Epigenetic factors also regulate behavioral functions. ${ }^{24}$

Chomsky uses the term "genetic endowment." But he is also one of the few linguists to acknowledge that working out the process of genetically fixing the values 
of linguistic primitives is a far from trivial task. Spelling out the right version of innateness that underlies our ability to do language is an enormous challenge. Hornstein rightly suggested that linguists interested in meeting it would effectively be biologists, regardless of their success. ${ }^{25}$

\section{Faculty of Language in the Narrow Sense}

In 2002, Chomsky, Marc Hauser, and W. Tecumseh Fitch introduced a distinction between the language faculty in a narrow and broad sense (FLN and FLB, respectively). ${ }^{26}$ FLN was described as the "only uniquely human component" of the language faculty, while FLB included "a sensory-motor system, a conceptual-intentional system, and the computational mechanisms for recursion, providing the capacity to generate an infinite range of expressions from a finite set of elements." ${ }^{27}$ Although FLB is clearly defined, and some of its components are unambiguously identified, FLN is harder to pin down.

Chomsky, Hauser, and Fitch defined FLN as a component unique to human language, possibly consisting only of recursion. In 2005, they suggested that

[t]he contents of FLN are to be empirically determined, and could possibly be empty, if empirical findings showed that none of the mechanisms involved are uniquely human or unique to language, and that only the way they are integrated is specific to human language. ${ }^{28}$

In 2009, Fitch then argued that "FLN was intended to have a considerably narrower scope, perhaps even denoting an empty set, but has been read simply as 'language' by some and 'Universal Grammar' by others." ${ }^{29}$ This last view points to the terminological confusion that arose in the various readings of the term "FLN." This confusion is unfortunate because the original distinction was "intended as a terminological aid to interdisciplinary discussion and rapprochement." ${ }^{30}$

The identification of FLN and UG is wrong. If UG equals FLN, and if FLN is, indeed, an empty set-a possibility that Chomsky has once again left open in his latest book with Robert Berwick ${ }^{31}$-scholars outside generative linguistics would inevitably question the need to assume a UG-shaped form of innateness. Furthermore, if UG and FLN are indeed the same, why are two terms needed to denote one object?

But, of course, FLN is not the same thing as UG. FLN is unique to humans and unique to language. UG does not have this character. ${ }^{32} \mathrm{UG}$ is relevant to both FLN's language-specific properties, and FLB's non-language-specific properties. Clearly, FLN is not tantamount to UG. Any suggestions to the contrary represent a misuse of two important terms in linguistics. It is a mistake that many linguists have made. ${ }^{33}$

\section{Hardwired}

Aside from inborn reflexes, remarkably few abilities are hardwired in humans. ${ }^{34}$

Linguists have talked about hardwired $\mathrm{UG},{ }^{35}$ hardwired principles and parameters, ${ }^{36}$ hardwired syntactic categories, ${ }^{37}$ hardwired Merge, ${ }^{38}$ hardwired semantic relations, ${ }^{39}$ and hardwired systems of lexicalizable concepts. ${ }^{40}$ According to common assumptions, something hardwired is both pre-programmed and behaviorally inflexible. Is the development of our innate ability for language behaviorally inflexible? Do children develop language in a way that is not affected by the environment? Feral children do not receive linguistic input in infancy. What impedes their development of innate syntactic categories? How can we meaningfully talk about a critical period for first language acquisition if a great deal of linguistic machinery is hardwired and will be inexorably manifested?

Few linguistic concepts are hardwired. It is not entirely clear that the brain itself is hardwired. This is evident from the lack of consensus on a number of critical issues, such as the lifetime of neuronal spines. ${ }^{41}$

\section{The Metaphors of Language Development}

\section{In Aspects of the Theory of Syntax, Chomsky argued that}

[1]inguistic theory is concerned primarily with an ideal speaker-listener, in a completely homogeneous speech-community, who knows its language perfectly and is unaffected by such grammatically irrelevant conditions as memory limitations, distractions, shifts of attention and interest, and errors (random or characteristic) in applying his knowledge of the language in actual performance. ${ }^{42}$

In his later work, Chomsky introduced the notion of instantaneous acquisition. ${ }^{43}$ This is a metaphor for an idealized version of acquisition, one that abstracts away from actual developmental stages on the assumption that, barring extreme cases, these stages are uniform and have no impact on the acquired grammar. ${ }^{44}$ Chomsky took care to highlight that these are idealizations. ${ }^{45}$

Eventually, Chomsky's completely homogeneous speech community and ideal speaker became embodied in the idea that, absent a severe pathology, adult performance is essentially homogeneous. ${ }^{46}$ This is demonstrably false, since cognitive phenotypes are not always binary. Individuals with a pathogenic variant of a gene can be impaired in different ways. This may lead to different cognitive phenotypes, at times not even reaching a cutoff point where the diagnosis of a specific pathology can be made. ${ }^{47}$ Variation can be found in the absence of any pathology even among speakers of the same language, and even within a speaker past the acquisition period. 
Over the last few decades, some linguists converted these idealizations into facts. Consider the claim of linguist Guglielmo Cinque:

The problem of parameter setting is usually posed in the context of the simplifying assumption that acquisition is instantaneous (cf. Chomsky 1975, pp. 119-22). My point is that such an assumption may not be just a convenient (and innocuous) idealization. It may well prove to be the only pertinent way to approach the question. Considerations of the actual stages of acquisition ... run the risk of distorting matters. ${ }^{48}$

Cinque suggests that we can approach the question of language acquisition by focusing on primitives he takes to be innately available, while ignoring both the way acquisition unfolds and the role of the environment. The problem is that the metaphor of instantaneous acquisition assumes that innateness and the environment are fully separable. But innateness does not work alone because genes do not work alone. Development is rarely hardwired, ${ }^{49}$ and language acquisition cannot be an instantaneous, almost reflex-like realization of innate structures and primitives. The metaphors of language development were once useful. It is through their subsequent use that they became unhelpful. Catchy metaphors, of course, make for popular quotes that conveniently express half-truths. And it is for this reason that they should never be taken too seriously or treated as dogma. According to Norbert Wiener and Arturo Rosenblueth, "the price of metaphor is eternal vigilance." 50

\section{Grammaticality Judgment}

Despite being frequently used, "grammaticality judgment" is a problematic term. Some linguists have argued that acceptability and grammaticality are distinct $;{ }^{51}$ others have argued the reverse. Do the terms "acceptability" and "grammaticality" refer to the same thing? If they do, why don't we just settle on one term? If they do not, do speakers provide different kinds of answers when asked about the grammaticality or the acceptability of a sentence in their native language?

A grammatical sentence conforms to the rules of a given language. An acceptable sentence refers to a speaker's perception of the stimulus in terms of her own linguistic repertoire. ${ }^{52}$ There are sentences that are not acceptable or in use-but for reasons that have nothing to do with violating linguistic rules. The sentence, "That that that Bill left Mary amused Sam is interesting is sad," is a grammatical sentence of English. ${ }^{53}$ Native speakers would not produce it often and they would certainly not rate it as acceptable as the very similar, "It is sad that it is interesting that it amused Sam that Bill left Mary."54

If grammaticality also reflects cognitive biases, asking a speaker to provide grammaticality judgments means asking for judgments about the interactions of all linguistic and cognitive factors that determine the limits of grammar. No speaker can provide this. Nor can any linguist. The term "grammaticality judgments" has been correctly labelled a misnomer. Speakers have intuitions only about their perception of linguistic stimuli. ${ }^{55}$

\section{Bilingual Advantage}

The effort of mentally juggling two linguistic systems can be linked to better performance in certain cognitive tasks. ${ }^{56}$ Thus the bilingual advantage. The term itself is neither ambiguous, problematic, nor misused. But its use calls for caution. As various scholars tested an ever-growing number of populations in order to discover the magnitude of the bilingual advantage, it became clear that one can also talk about a bilingual disadvantage. ${ }^{57} \mathrm{~A}$ more useful term that emerged in the same literature is "trade-off." ${ }^{58} \mathrm{In}$ this sense, an advantage in one measure may bring along other effects, hence the term "bilingual advantage" might only provide half of the picture with respect to bilingual effects on cognition. Talking about the overall impact or effect of bilingualism on cognition may come closer to acknowledging the surrounding implications.

\section{Optimal Design and Perfect System}

Hauser, Chomsky, and Fitch suggest that FLN can be thought of as "a kind of 'optimal solution' to the problem of linking the sensory-motor and conceptual-intentional systems, ${ }^{59}$ and of satisfying the interface conditions between FLN and FLB. This view is also present in Chomsky's earlier work under the name "strong minimalist thesis" (SMT). ${ }^{60}$ In other work, the language system has been described as perfect. ${ }^{61}$ These notions are elusive. Most scholars understand them as denoting principles of parsimony, economy, and elegance, ${ }^{62}$ but the way these properties are attributed to language is unclear. Do parsimony, economy, and elegance characterize language, or theories about language? ${ }^{63}$

Language develops some of its characteristics by adaptation to environmental triggers. There is evidence that over time, speakers develop effort-accuracy trade-offs. ${ }^{64}$ Languages are also reshaped to become more learnable..$^{65}$ One might say that the language system is becoming more optimal in response to adaptation pressures. This trend toward efficiency is probably not due to the language system itself. The human mind has been described as making optimal use of its cognitive resources across domains ${ }^{66}$ It is unclear whether the optimality of language is due to the recruitment of general principles of rationality and adaptation, or whether language, possibly by being unique at some level, stands out from other cognitive modules.

It is harder to argue that all linguistic theories are optimal, especially in relation to the preceding suggestions 
that innateness consists of an unknown, ever-growing number of features, parameters, and other primitives.

\section{Taking Stock}

The objective of this essay is to attain a higher level of terminological clarity and coherence within the field of linguistics. If successful, this process may help improve its visibility in neighboring fields, such as other parts of psychology, biology, and neuroscience. This visibility is, in fact, extremely important. As scientists of language, linguists should offer robust and cohesive theories and this is particularly true for claims about innateness, which must make sense from a biological point of view. Some months ago, I attended a conference that dealt with certain linguistic primitives. A talk was given by a senior linguist who argued for the removal of a specific primitive from UG. UG was mentioned at the beginning of the talk and never referred to again. Nor was it explained why the feature was allocated to UG in the first place. UG, it should be stressed, is defined as our biological endowment for language, and claims made about UG in linguistics must be plausible in biology. I asked the speaker how the feature in question came to be part of UG. I was told that this had been a common assumption among theoretical linguists since the 1970s. I then asked how theories of UG might support such a claim. I found it hard to see how a biologist would be convinced that the biological endowment for language encodes all of these grammatical features. Defending our theories from interdisciplinary criticisms, I suggested, might be extremely difficult. To my surprise, the speaker responded that he had no interest in interdisciplinary work. These considerations, I realized, simply did not enter the picture. For the speaker, UG is simply a repository of linguistic primitives that can be disconnected from human biology.

This is why terminological clarity matters.

Evelina Leivada is a psycholinguist at the Universitat Rovira $i$ Virgili in Tarragona, Spain.

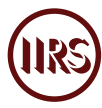

1. Remi van Trijp, "Use Your Own Toothbrush," Essays in Linguistics (blog).

2. Peter Hagoort, "Linguistics quo vadis? An Outsider Perspective," talk presented at SLE 2014: 47th Annual Meeting of the Societas Linguistica Europaea, Adam Mickiewicz University, Poznań, Poland, 2014.

3. Tanja Kupisch and Jason Rothman, "Terminology Matters! Why Difference Is Not Incompleteness and How Early Child Bilinguals Are Heritage Speakers," Inter- national Journal of Bilingualism 22, no. 5 (2016): 574, doi:10.1177/1367006916654355.

4. Scott Lilienfeld et al., "Fifty Psychological and Psychiatric Terms to Avoid: A List of Inaccurate, Misleading, Misused, Ambiguous, and Logically Confused Words and Phrases,", Frontiers in Psychology 6 (2015): 1,100, doi:10.3389/ fpsyg.2015.01100; Scott Lilienfeld et al., "50 Differences That Make a Difference: A Compendium of Frequently Confused Term Pairs in Psychology," Frontiers in Education 2 (2017): 37, doi:10.3389/feduc.2017.00037.

5. Take any issue of a journal dedicated to theoretical or experimental generative linguistics and check how many articles in that issue do not feature any of the aforementioned terms. The answer is likely very few, or none. Each article will probably mention more than one of these terms on multiple occasions. The terms discussed here are not some barely encountered notions, the status of which is of minor relevance to most of the field of linguistics.

6. Noam Chomsky, Lectures on Government and Binding (Dordrecht: Foris, 1981).

7. Noam Chomsky, "On the Representation of Form and Function," The Linguistic Review 1, no. 1 (1981): 3-40, doi:10.1515/ tlir.1981.1.1.3.

8. José-Luis Mendívil-Giró, "Is Universal Grammar Ready for Retirement? A Short Review of a Longstanding Misinterpretation," Journal of Linguistics 54, no. 4 (2018): 861, doi:10.1017/s0022226718000166.

9. I thank Norbert Hornstein for this point. For useful feedback, I thank Antonio Benítez-Burraco, Antonio Fábregas, Kleanthes Grohmann, Norbert Hornstein, Myrtani Pieri, Jason Rothman, and Patrick Trettenbrein.

10. Almost $80 \%$ of the world's languages surveyed in the World Atlas of Language Structures agree with this pattern. See Matthew Dryer, "Order of Adjective and Noun," in The World Atlas of Language Structures Online, eds. Matthew Dryer and Martin Haspelmath (Leipzig: Max Planck Institute for Evolutionary Anthropology, 2008).

11. Jennifer Culbertson, Paul Smolensky, and Géraldine Legendre, "Learning Biases Predict a Word Order Universal," Cognition 122, no. 3 (2012): 306-29, doi:10.1016/j.cognition.2011.10.017.

12. Noam Chomsky, "Three Factors in Language Design," Linguistic Inquiry 36, no. 1 (2005): 1-22, doi:10.1162/0024389052993655.

13. Janet Dean Fodor, "Setting Syntactic Parameters," in The Handbook of Contemporary Syntactic Theory, eds. Mark Baltin and Chris Collins (Malden \& Oxford: Blackwell, 2001), 734, doi:10.1002/9780470756416.ch23.

14. Frederick Newmeyer, Possible and Probable Languages: A Generative Perspective on Linguistic Typology (Oxford: Oxford University Press, 2005), 83.

15. Some linguists, especially within the cartographic framework, have supported the following equation: one (morphosyntactic) property-one feature-one designated slot in the innate functional hierarchy. See, for example, 
Guglielmo Cinque and Luigi Rizzi, "The Cartography of Syntactic Structures," in Studies in Linguistics: CISCL Working Papers on Language and Cognition, ed. Vincenzo Moscati (Siena: University of Siena, 2008), 5:44. As a result, incredibly rich and ever-growing arrays of syntactic heads have been cast as innate on the basis of variant morphosyntactic realizations.

16. Greville Corbett, "Features: Essential Notions," in Features: Perspectives on a Key Notion in Linguistics, eds. Anna Kibort and Greville Corbett (Oxford: Oxford University Press, 2010), 18.

17. Extended projection principle: A linguistic constraint that suggests that all clauses must have a subject. See Chomsky, Lectures on Government and Binding.

18. Ur Shlonsky, "The Cartographic Enterprise in Syntax," Language and Linguistics Compass 4, no. 6 (2010): 424, doi:10.1111/j.1749-818x.2010.00202.x.

19. The logic of the argument must be clear: How can we explain structure $B$ in language $C$ ? An innate primitive must be behind it-call it feature/parameter A. And what reason do we have to believe that A exists? Structure B. In a similar context, Bridget Samuels has noted that

upon observing that a certain language has no consonant clusters, one posits that these facts are not random, but are due to a constraint like ${ }^{*} \mathrm{CC}$. But then, when one asks why the language has no consonant clusters, the answer is because ${ }^{*} \mathrm{CC}$ bans them. While perhaps on some level all logic is ultimately circular, this is a very small circle.

See Bridget Samuels, "Biolinguistics in Phonology: A Prospectus," Phonological Studies 18, (2015): 164.

20. Denis Bouchard, "Solving the UG Problem," Biolinguistics 6, no. 1 (2012): 12.

21. David Adger and Peter Svenonius, "Features in Minimalist Syntax," in The Oxford Handbook of Linguistic Minimalism, ed. Cedric Boeckx (Oxford: Oxford University Press, 2011), 28.

22. It is important that this terminological fluidity is noted in order to avoid talking about the same thing with very different underlying assumptions in mind. Linguists have recognized field-fragmentation as a potential danger for coherence and taken steps in this direction. A good example is the conference "Generative Syntax in the Twenty-First Century: The Road Ahead" that took place in Athens in 2015. Among the challenges that were addressed in this event was the coherence of the field. As suggested during the call,

[g]iven the large number of different analytic approaches, it has resulted in small groups working on $\mathrm{x}, \mathrm{y}$, or z. From a scientific point of view, this is not problematic, but it raises difficulties when it comes to interaction, funding, recruitment and external visibility.

The present work has a similar purpose in registering a different danger: if different groups work on $\mathrm{x}$ but use $\mathrm{x}$ to refer to different things, this state of affairs raises even more difficulties in relation to coherence, interaction, and external visibility.

23. Ana Villar and Antoni Gomila, "A Minor Role for Genetics in Language Evolution," Journal of Anthropological Sciences 91 (2013): 31.

24. Cedric Boeckx and Evelina Leivada, "On the Particulars of Universal Grammar: Implications for Acquisition," Language Sciences 46 (2014): 189-98, doi:10.1016/j.langsci.2014.03.004; Antonio Benítez-Burraco, "Problematic Aspects of the Genetic Analysis of the Specific Disorders of the Language: FOXP2 as Paradigm," Neurología 27, no. 4 (2012): 225-33, doi:10.1016/j.nrleng.2012.05.005.

25. Norbert Hornstein, "How Biological Is Biolinguistics?" Faculty of Language, April 27, 2017.

26. Marc Hauser, Noam Chomsky, and W. Tecumseh Fitch, "The Faculty of Language: What Is It, Who Has It, and How Did It Evolve?" Science 298, no. 5,598 (2002): 1,569-79, doi:10.1126/ science.298.5598.1569.

27. Hauser, Chomsky, and Fitch, "The Faculty of Language," 1,569 .

28. W. Tecumseh Fitch, Marc Hauser, and Noam Chomsky, "The Evolution of the Language Faculty: Clarifications and Implications," Cognition 97, no. 2 (2005): 181, doi:10.1016/j. cognition.2005.02.005.

29. W. Tecumseh Fitch, "Prolegomena to a Future Science of Biolinguistics,” Biolinguistics 3, no. 4 (2009): 288.

30. Fitch, Hauser, and Chomsky, "Evolution of the Language Faculty," 181.

31. More specifically, Berwick and Chomsky suggest that humans, but no other animal, have the ability to construct unbounded arrays of hierarchically structured expressions through Merge. Yet they also highlight that this ability may well be implemented by pre-existing, non-language-specific, repurposed wetware. The use of already existing machinery implies that at some level the uniqueness of this ability does not hold, by means of being constrained by non-language-specific biophysical factors. See Robert Berwick and Noam Chomsky, Why Only Us: Language and Evolution (Cambridge: MIT Press, 2016), 132.

32. Patrick Trettenbrein, "The 'Grammar' in Universal Grammar: A Biolinguistic Clarification," Questions and Answers in Linguistics 2, no. 1 (2015): 4, doi:10.1515/qal-2015-0005.

33. See, for example, Halldór Ármann Sigurðsson, "On UG and Materialization,” Linguistic Analysis 37 (2011): 371; Frederick Newmeyer, "Goals and Methods of Generative Syntax," in The Cambridge Handbook of Generative Syntax, ed. Marcel den Dikken (Cambridge: Cambridge University Press, 2013), 72.

34. Lilienfeld et al., "Fifty Psychological and Psychiatric Terms."

35. Trettenbrein, "The 'Grammar' in Universal Grammar," 3.

36. Richard Kayne, "Antisymmetry and the Lexicon," in The Biolinguistic Enterprise: New Perspectives on the Evolution and Nature of the Human Language Faculty, eds. Anna Maria Di Sciullo and Cedric Boeckx (Oxford: Oxford University Press, 2011), 334. 
37. Frank Wijnen, "Acquisition of Linguistic Categories: Cross-Domain Convergences," in Birdsong, Speech, and Language: Exploring the Evolution of Mind and Brain, eds. Johan Bolhuis and Martin Everaert (Cambridge: MIT Press, 2013), 157.

38. Peter Svenonius, "Linguistic Universals and the Category P," September 16, 2005, 1.

39. Rasha Abdel Rahman and Alissa Melinger, "Dismissing Lexical Competition Does Not Make Speaking Any Easier: A Rejoinder to Mahon and Caramazza (2009)," Language and Cognitive Processes 24, no. 5 (2009): 753, doi:10.1080/01690960802648491.

40. Wendy Francis, "Bilingual Semantic and Conceptual Representation," in Handbook of Bilingualism: Psycholinguistic Approaches, eds. Judith Kroll and Annette de Groot (Oxford: Oxford University Press, 2005), 251.

41. Ole Ottersen and P. Johannes Helm, "How Hardwired Is the Brain?" Nature 420, no. 6,917 (2002): 752, doi:10.1038/420751a.

42. Noam Chomsky, Aspects of the Theory of Syntax (Cambridge: MIT Press, 1965), 3.

43. Noam Chomsky, Reflections on Language (New York: Pantheon, 1975), 119.

44. Dalila Ayoun, Parameter Setting in Language Acquisition (London: Continuum, 2003), 7.

45. Noam Chomsky, Aspects, 4.

46. Stephen Anderson and David Lightfoot, "The Human Language Faculty as an Organ," Annual Review of Physiology 62, no. 1 (2000): 698, doi:10.1146/annurev.physiol.62.1.697.

47. For further discussion on this point, see Kleanthes Grohmann and Evelina Leivada, "Reconciling Linguistic Theories on Comparative Variation with an Evolutionarily Plausible Language Faculty," in A Festschrift for Ian Roberts, eds. András Bárány et al. (Berlin: Language Science Press, in press).

48. Guglielmo Cinque, "Parameter Setting in 'Instantaneous' and Real-Time Acquisition," Behavioral and Brain Sciences 12, no. 2 (1989): 336, doi:10.1017/s0140525x00048913.

49. Gregory Wray, "Evolution and Development," in Evolution: The First Four Billion Years, eds. Michael Ruse and Joseph Travis (Cambridge: Harvard University Press, 2009), 228.

50. Richard Lewontin, "In the Beginning Was the Word," Science 291, no. 5,507 (2001): 1,264, doi:10.1126/science.1057124.

51. Noam Chomsky, Aspects, 11.

52. Ellen Gurman Bard, Dan Robertson, and Antonella Sorace, "Magnitude Estimation of Linguistic Acceptability," Language 72, no. 1 (1996): 33, doi:10.2307/416793.

53. The example is from Norbert Hornstein, "Acceptability and Grammaticality," Faculty of Language, February 22, 2013.

54. This is not due to the fact that a linguistic rule is violated. The speakers' effort to parse the first sentence would be significantly strained upon encountering the third 'that,' due to a cognitive bias to provide more attentional resources to novel information. The bias is called novel information bias and can be thought of as the cognitive underpinning of identity avoidance theories in linguistics. See Evelina Leivada, "What's in (a) Label? Neural Origins and Behavioral Manifestations of Identity Avoidance in Language and Cognition,” Biolinguistics 11, no. SI (2017): 221-50.

55. Cedric Boeckx, Language in Cognition: Uncovering Mental Structures and the Rules behind Them (Maden: Wiley-Blackwell, 2010), 138.

56. Some selected references follow: Ellen Bialystok, Bilingualism in Development: Language, Literacy, and Cognition (New York: Cambridge University Press, 2001); Ellen Bialystok et al., "Bilingualism, Aging, and Cognitive Control: Evidence from the Simon Task," Psychology and Aging 19, no. 2 (2004): 290-303, doi:10.1037/0882-7974.19.2.290; Gigi Luk et al., "Lifelong Bilingualism Maintains White Matter Integrity in Older Adults," Journal of Neuroscience 31, no. 46 (2011): 16,808-13, doi:10.1523/JNEUROSCI.4563-11.2011; Vincent DeLuca et al., "Redefining Bilingualism as a Spectrum of Experiences that Differentially Affects Brain Structure and Function," Proceedings of the National Academy of Sciences 116, no. 15 (2019): 7,565-74, doi:10.1073/pnas.1811513116.

57. Elin Runnqvist et al., "A Disadvantage in Bilingual Sentence Production Modulated by Syntactic Frequency and Similarity across Languages," Cognition 129, no. 2 (2013): 256-63, doi:10.1016/j.cognition.2013.07.008.

58. Esli Struys, Wouter Duyck, and Evy Woumans, "The Role of Cognitive Development and Strategic Task Tendencies in the Bilingual Advantage Controversy," Frontiers in Psychology 9 (2018): 2, doi:10.3389/fpsyg.2018.01790.

59. Hauser, Chomsky, and Fitch, "The Faculty of Language," 1,574.

60. Noam Chomsky, "Derivation by Phase," in Ken Hale: A Life in Language, ed. Michael Kenstowicz (Cambridge: MIT Press, 2001), 1.

61. Noam Chomsky, On Nature and Language (Cambridge: Cambridge University Press, 2002), 58. Koji Fujita, "Facing the Logical Problem of Language Evolution," English Linguistics 24 (2007): 78, doi:10.9793/elsj1984.24.78.

62. Fahad Rashed Al-Mutairi, The Minimalist Program: The Nature and Plausibility of Chomsky's Biolinguistics (Cambridge: Cambridge University Press, 2014), 34, and references therein.

63. In relation to the second reading, the following claim by Cedric Boeckx is quite illuminating:

The minimalist program for linguistic theory adopts as its working hypothesis the idea that Universal Grammar is "perfectly" designed, that is, it contains nothing more than what follows from our best guesses regarding conceptual, biological, physical necessity [emphases added]. This hypothesis is probably too strong (Chomsky calls it the "strong minimalist thesis"), but in practice scientists often adopt the strongest possible thesis as their working hypothesis. The strongest hypothesis then acts as a limiting case, enabling us to see more precisely where and when the hypothesis fails and how much of it may be true. The strong minimalist thesis is indeed a wild idea. 
Cedric Boeckx, Linguistic Minimalism: Origins, Concepts, Methods, and Aims (Oxford: Oxford University Press, 2006), 4.

64. George Zipf, Human Behavior and the Principle of Least Effort (Cambridge, MA: Addison-Wesley, 1949).

65. Simon Kirby, Hannah Cornish, and Kenny Smith, "Cumulative Cultural Evolution in the Laboratory: An Experimental Approach to the Origins of Structure in Human Language,",
Proceedings of the National Academy of Sciences 105, no. 31 (2008): 10,681-6, doi:10.1073/pnas.0707835105.

66. Falk Lieder and Thomas Griffiths, "Resource-Rational Analysis: Understanding Human Cognition as the Optimal Use of Limited Computational Resources," Behavioral and Brain Sciences 43 (2020): 1-85, doi:10.1017/s0140525x1900061x. 